\title{
Incentivizing stable path selection in future Internet architectures
}

\section{Conference Paper}

Author(s):

Scherrer, Simon; Legner, Markus (D); Perrig, Adrian; Schmid, Stefan

Publication date:

2020-12

Permanent link:

https://doi.org/10.3929/ethz-b-000452804

\section{Rights / license:}

Creative Commons Attribution 4.0 International

\section{Originally published in:}

Performance evaluation 144, https://doi.org/10.1016/j.peva.2020.102137

\section{Funding acknowledgement:}

182005 - ESCALATE: Efficient and Scalable Algorithms for Large Flow Detection (SNF) 


\title{
Incentivizing stable path selection in future Internet architectures
}

\author{
Simon Scherrer ${ }^{\mathrm{a}, *}$, Markus Legner ${ }^{\mathrm{a}}$, Adrian Perrig ${ }^{\mathrm{a}}$, Stefan Schmid ${ }^{\mathrm{b}}$ \\ a Department of Computer Science, ETH Zurich, Zurich, Switzerland \\ ${ }^{\mathrm{b}}$ Faculty of Computer Science, University of Vienna, Vienna, Austria
}

\section{A R T I C L E I N F O}

\section{Article history:}

Available online 1 October 2020

\section{Keywords:}

Game Theory

Future Internet

Path Selection

\begin{abstract}
A B S T R A C T
By delegating path control to end-hosts, future Internet architectures offer flexibility for path selection. However, there is a concern that the distributed routing decisions by end-hosts, in particular load-adaptive routing, can lead to oscillations if path selection is performed without coordination or accurate load information. Prior research has addressed this problem by devising path-selection policies that lead to stability. However, little is known about the viability of these policies in the Internet context, where selfish end-hosts can deviate from a prescribed policy if such a deviation is beneficial from their individual perspective. In order to achieve network stability in future Internet architectures, it is essential that end-hosts have an incentive to adopt a stability-oriented path-selection policy.

In this work, we perform the first incentive analysis of the stability-inducing pathselection policies proposed in the literature. Building on a game-theoretic model of end-host path selection, we show that these policies are in fact incompatible with the self-interest of end-hosts, as these strategies make it worthwhile to pursue an oscillatory path-selection strategy. Therefore, stability in networks with selfish end-hosts must be enforced by incentive-compatible mechanisms. We present two such mechanisms and formally prove their incentive compatibility.
\end{abstract}

(C) 2020 The Author(s). Published by Elsevier B.V. This is an open access article under the CC BY license (http://creativecommons.org/licenses/by/4.0/).

\section{Introduction}

The past 20 years of research on next-generation Internet architectures have shown the benefits of path awareness and path control for end-hosts, and multiple path-aware network architectures have been proposed. Many of these architectures, including RON [1], Platypus [2], MIRO [3], Pathlets [4], Segment Routing [5], and SCION [6], allow end-hosts to select the inter-domain paths over which their data packets are forwarded. One principal argument for such path control is that it enables load-adaptive routing, i.e., allows the end-hosts to avoid congested links, and should therefore lead to a relatively even traffic distribution. However, load-adaptive routing creates new challenges, in particular the introduction of instabilities under certain conditions. Instability due to load-adaptive routing typically appears in the form of oscillations, i.e., periodic up- and downswings of link utilization, leading to a large variance of the traffic load in a short time span. According to the IETF, a central obstacle to deployment of path-aware network architectures is 'oscillations based on feedback loops, as hosts move from path to path' [7]. Indeed, such oscillations can be shown to occur if path-selection decisions are taken on the basis of outdated load information [8,9], which is the case in any real system.

\footnotetext{
* Corresponding author.

E-mail address: simon.scherrer@inf.ethz.ch (S. Scherrer).
} 
Such oscillations are undesirable for many reasons, both from the perspective of the end-hosts and the perspective of the network operator. If oscillation occurs when a link is near its capacity limit, there is a danger of queue buildup, jitter, and, as a result, unpredictable performance. Moreover, oscillation temporarily leads to a heavily skewed load distribution over paths, causing higher overall queuing latency than with a more equal traffic distribution. Due to the large variance of the load level over time, network operators have to perform substantial overprovisioning of link capacities, which is undesirable from a business perspective. Moreover, oscillation of inter-domain traffic imposes additional overhead for intra-domain traffic engineering (e.g., MPLS circuit setup), as oscillating inter-domain flows may constantly switch between inter-AS interfaces. From the end-host perspective, oscillation causes packet loss and thus forces the congestion-control algorithms to recurring restarts, negatively affecting throughput.

To avoid these damaging effects, researchers have devised numerous schemes that aim to guarantee stability of loadadaptive routing. However, to the best of our knowledge, no scheme so far has aimed at providing stability in Internet architectures with end-host path control. Many systems have been designed under the assumption of network-based path selection, i.e., hop-by-hop forwarding according to decisions taken by intermediate routers [10-13]. These systems achieve convergence by appropriately adjusting how much traffic is forwarded to each next hop towards a destination and cannot be used if packets must be sent along paths selected by end-hosts. Other systems allow end-point path selection, but are targeted to an intra-domain context where the end-points (typically ingress and egress routers) are under the control of a network operator $[8,14-18]$. In an intra-domain context, network operators are able to prescribe arbitrary path-selection procedures that generate stability. Conversely, in an inter-domain context, the end-points are not under control of network operators and can thus not be forced to adopt a non-oscillatory path-selection strategy. Instead, as end-hosts must be assumed to be selfish, they can only be expected to adopt path-selection strategies that optimize performance from their individual perspective.

By performing a game-theoretic analysis, we show in this paper that the non-oscillatory path-selection strategies traditionally proposed in the literature on stable source routing $[8,14-18]$ are incompatible with the self-interest of endhosts. Assuming that such non-oscillatory path-selection strategies are universally adopted, an end-host can increase its utility by deviating in favor of a strategy that is oscillatory. Therefore, stability of load-adaptive routing in an interdomain context cannot be achieved by relying only on end-point path selection. Instead, network operators have to incentivize end-hosts to adopt one of the well-known convergent path-selection strategies with stabilization mechanisms. These mechanisms have to be incentive-compatible, i.e., the mechanisms must create an incentive structure such that it is in an end-host's self-interest to adopt a non-oscillatory path-selection strategy. In this work, we present two such stabilization mechanisms, FLOSS and CROSS, and formally prove their incentive compatibility. These mechanisms employ different techniques to disincentivize oscillatory switching between paths, namely limiting the migration rate between paths (FLOSS) and imposing a cost on switching between paths (CROSS). To complement our mainly theoretical work, we also discuss how our findings could be practically applied.

\subsection{Contribution}

This paper revisits the theoretical study of the dynamic effects of end-point path selection, for the first time focusing the analysis on inter-domain networks where the end-points are selfish and uncontrolled. We present a game-theoretic model that allows us to investigate which path-selection strategies will be adopted by selfish end-hosts. In particular, we introduce the notion of equilibria to path-selection strategies (PSS equilibria). Moreover, we formally show that the non-oscillatory path-selection strategies proposed in the existing literature do not form such PSS equilibria. Thus, we provide evidence towards the hypothesis that stability in load-adaptive routing over multiple domains cannot be achieved by exclusively relying on end-hosts' path-selection behavior. To remedy this problem, we leverage insights from mechanism design to devise two incentive-compatible stabilization mechanisms enforced by network operators. While these mechanisms build on existing insights from intra-domain traffic engineering, their methods of incentivization represent a novel approach to achieve stability in inter-domain networks with load-adaptive routing. We formally prove the incentive compatibility of both mechanisms and discuss their practical application.

\section{Oscillation model}

\subsection{Parallel-path systems}

In order to study oscillation in network architectures with end-host path selection, we build on the well-established Wardrop model [19], which is the standard model for studying the interactions of selfish agents in computer networks [20-22]. In the Wardrop model, an infinite number of end-hosts, each controlling an infinitesimal traffic share, select one path $\pi$ among multiple paths $\Pi$ between two network nodes. Every path $\pi$ has a load-dependent cost, where the path-cost function $c_{\pi}$ is typically interpreted as latency. The end-hosts' path-selection decisions form a congestion game, where the path-selection decisions of end-hosts both determine and follow the load $f_{\pi}$ on every path $\pi$ [23-25].

In this work, we analyze congestion games with a temporal component, i.e., end-hosts take path-selection decisions over time based on currently available information. More precisely, an end-host performs an average of $r>0$ reevaluations per unit of time. The aggregate re-evaluation behavior is uniform over time, i.e., when dividing time into intervals of length $\epsilon \in(0,1], r \epsilon$ re-evaluations are performed in any interval 
Whenever an end-host performs a re-evaluation, it chooses one path $\pi$ to its destination according to a freely chosen path-selection strategy $\sigma$. We thus formalize the environment of congestion games as parallel-path systems:

Definition 1. A parallel-path system $O:=\left(\Pi, r, p, T, A_{0}, v\right)$ is a tuple, where a total demand normalized to 1 is distributed over parallel paths $\pi \in \Pi$ among which end-hosts can select; $r>0$ is the average number of re-evaluations per end-host and unit of time; $p \geq 1$ is the steepness of the path cost as a function of the load (i.e., $\left.c_{\pi}=\left(f_{\pi}\right)^{p}\right) ; T \geq 0$ is the average time that it takes for cost information to reach the agents; $\mathbf{A}_{\mathbf{0}} \in[0,1]^{|\Pi|}$ is the initial load matrix, where the entry $\mathbf{A}_{\mathbf{0}_{\pi}}=f_{\pi}(0)$; and $v$ is the strategy profile, defining for every available path-selection strategy $\sigma$ the share $v(\sigma)$ of end-hosts that permanently apply strategy $\sigma$.

Every congestion game possesses at least one Wardrop equilibrium, consisting of a traffic distribution where no single agent can reduce its cost by selecting an alternative path [25]. If the agents take path-selection decisions based on up-todate cost information of paths $(T=0)$, convergence to Wardrop equilibria is guaranteed and persistent oscillations can thus not arise [26-28]. However, in practice, the cost information possessed by agents is stale $(T>0)$, i.e., the information describes an older state of the network. If such stale information is present, undesirable oscillations can arise [8]. Therefore, parallel-path systems can be oscillation-prone:

\section{Definition 2. A parallel-path system $O$ is oscillation-prone iff $T>0$.}

In this work, we study oscillation-prone systems with two paths $\alpha$ and $\beta$ (i.e., $|\Pi|=2$ ), but our insights directly generalize to more paths. Due to total demand normalization, it holds that $f_{\beta}(t)=1-f_{\alpha}(t)$ for all $t \geq 0$. Thus, the unique Wardrop equilibrium in a two-path oscillation-prone system is given by $f_{\alpha}=f_{\beta}=1 / 2$. Moreover, we assume w.l.o.g. that the initial imbalance $A_{0}$ exists with the higher load on path $\alpha: f_{\alpha}(0)=A_{0}=\mathbf{A}_{\mathbf{0}_{\alpha}}>1 / 2$. For this system of two parallel paths, $\tilde{\pi}$ denotes the respective other path, i.e., $\tilde{\alpha}=\beta$ and $\tilde{\beta}=\alpha$.

Having introduced the concept of oscillation-prone systems, we next define notions of oscillation and stability. First, an oscillation-prone system experiences oscillation if the traffic distribution does not eventually become static:

Definition 3. An oscillation-prone system $O$ experiences oscillation if there exists no limit $\Delta^{*}$ of the function $\Delta(t)=$ $\left|f_{\alpha}(t)-f_{\beta}(t)\right|$ for $t \rightarrow \infty$.

Conversely, we understand stability simply as the absence of oscillation, i.e., stability is given if a limit $\Delta^{*}$ exists. However, to ensure optimal network utilization, the desirable state of the network is not only stability, but stability at equal load as given by the Wardrop equilibrium:

Definition 4. An oscillation-prone system $O$ is stable at equal load if

$$
\Delta^{*}:=\lim _{t \rightarrow \infty} \Delta(t)=0
$$

\subsection{Path-selection strategies}

In a congestion game, end-hosts select paths according to freely adopted path-selection strategies. In order to enable a theoretical treatment, we follow Fischer and Vöcking [8] in assuming that path-selection strategies are memory-less, i.e., not dependent on anything else than currently observable information. Therefore, any path-selection strategy $\sigma$ can be fully characterized by two elements, $\sigma=(R, u)$, which we will describe in the following.

First, every strategy is characterized by the expected time $R$ between re-evaluations of an end-host. The expected re-evaluation period $R$ reflects the reallocation behavior of end-hosts that non-deterministically re-evaluate the costs of path options, decide for one option based on the perceived costs, and keep sending on the selected path until the next re-evaluation is due. The expected re-evaluation period $R$ has to be in accordance with the parameter $r$ of the parallel-path system, which describes the average number of re-evaluations per end-host and unit of time. Hence, $R=1 / r$.

Second, every strategy $\sigma$ is based on a path-selection function $u\left(\pi, t \mid \pi^{\prime}\right)$, which gives the probability for selecting path $\pi$ at time $t$ if the currently used path is $\pi^{\prime}$. Given universal adoption of a strategy $\sigma$ and $r \epsilon$ re-evaluations per interval of length $\epsilon$, the number of end-hosts on path $\pi$ changes by the amount $\Delta_{\epsilon} f_{\pi}(t)=-r \epsilon \cdot u(\tilde{\pi}, t \mid \pi) \cdot f_{\pi}(t)+r \epsilon \cdot u(\pi, t \mid \tilde{\pi}) \cdot f_{\tilde{\pi}}(t)$ within an interval starting at time $t$, given a two-path system. If $\epsilon$ is chosen to be infinitesimal, we obtain the rate of change:

$$
\frac{\partial f_{\pi}(t)}{\partial t}=\lim _{\epsilon \rightarrow 0} \frac{\Delta_{\epsilon} f_{\pi}(t)}{\epsilon}=-r \cdot u(\tilde{\pi}, t \mid \pi) \cdot f_{\pi}(t)+r \cdot u(\pi, t \mid \tilde{\pi}) \cdot f_{\tilde{\pi}}(t)
$$

Throughout the rest of the paper, we describe oscillation dynamics by such differential equations.

An example of a path-selection strategy is the greedy path-selection strategy $\sigma_{\mathrm{g}}$, which selects the path perceived as cheaper:

$$
u_{\mathrm{g}}(\pi, t \mid \tilde{\pi})= \begin{cases}1 & \text { if } c_{\pi}(t-T)<c_{\tilde{\pi}}(t-T) \\ 0 & \text { otherwise }\end{cases}
$$




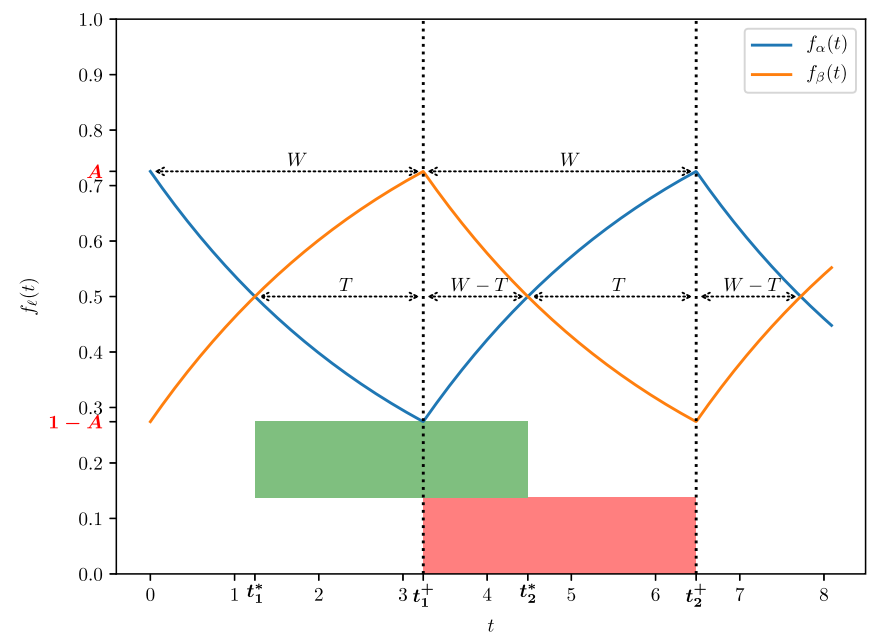

Fig. 1. Oscillation structure for oscillation-prone system $O=\left(\{\alpha, \beta\}, r=0.3, p \geq 1, T=2, A_{0}=A, v=\left\{\sigma_{\mathrm{g}} \mapsto 1\right\}\right)$. A and $W$ are calculated according to Eq. (6).

Conversely, the probability of staying on a path is $u_{\mathrm{g}}(\tilde{\pi}, t \mid \tilde{\pi})=1-u_{\mathrm{g}}(\pi, t \mid \tilde{\pi})$. At time $t$, the number of end-hosts on a more expensive path $\pi$ thus changes with rate $-r \cdot f_{\pi}(t)$.

Whether an oscillation-prone system in fact experiences oscillation entirely depends on the path-selection strategies adopted by end-hosts. In the next section, we present the example of an oscillation-prone system that experiences oscillation for some path-selection strategy, but converges to stability for a different strategy.

\subsection{Example of oscillation}

For every $T>0$, oscillation occurs in a system in which all agents adopt a greedy path-selection strategy $\sigma_{\mathrm{g}}$ presented in the previous section. The dynamics of a system with universal adoption of the greedy strategy are given by the partial differential equation ${ }^{1}$ :

$$
\frac{\partial f_{\alpha}(t)}{\partial t}= \begin{cases}-r \cdot f_{\alpha}(t) & \text { if } c_{\alpha}(t-T)>c_{\beta}(t-T) \\ r \cdot f_{\beta}(t) & \text { if } c_{\alpha}(t-T)<c_{\beta}(t-T) \\ 0 & \text { otherwise }\end{cases}
$$

We henceforth refer to turning points as all points in time $t^{+}$where $c_{\alpha}\left(t^{+}-T\right)=c_{\beta}\left(t^{+}-T\right)$, as $f_{\alpha}(t)$ switches between increasing and decreasing at these moments, and write $t^{+}(t)$ for the most recent turning point $t^{+}<t$.

Solving the differential equation piece-wise yields the following recursive function ${ }^{2}$ :

$$
f_{\alpha}(t)= \begin{cases}e^{-r \cdot\left(t-t^{+}(t)\right)} \cdot f_{\alpha}\left(t^{+}(t)\right) & \text { if } f_{\alpha}(t-T) \geq \frac{1}{2} \\ 1-e^{-r \cdot\left(t-t^{+}(t)\right)} \cdot f_{\beta}\left(t^{+}(t)\right) & \text { otherwise }\end{cases}
$$

Since $T$ is constant, $f_{\alpha}(t)$ is periodic after the first turning point $t_{1}^{+}$irrespective of the initial imbalance $A_{0}$. Therefore, the oscillation can be described by the non-recursive function:

$$
f_{\alpha}(t)= \begin{cases}e^{-r \cdot\left(t-t^{+}(t)\right)} \cdot A & \text { if } \frac{t^{+}(t)}{W} \text { is even, } \\ 1-e^{-r \cdot\left(t-t^{+}(t)\right)} \cdot A & \text { otherwise, }\end{cases}
$$

where

$$
W=\frac{\ln \left(2 e^{r T}-1\right)}{r}, \quad A=1-\frac{1}{2 e^{r T}},
$$

and $t^{+}(t)=t-(t \bmod W)$ is a multiple of $W$. Fig. 1 shows an example of $f_{\alpha}(t)$ for the oscillation-prone system $O=\left(\Pi=\{\alpha, \beta\}, r=0.3, p \geq 1, T=2, A_{0}=A, v=\left\{\sigma_{\mathrm{g}} \mapsto 1\right\}\right)$, where $A_{0}$ has been chosen as $A$ in order to skip the irregular starting phase. Fig. 1 also highlights the time interval during which path $\alpha$ is the cheaper path (in green, between $t_{1}^{*}$ and $t_{2}^{*}$ ) and the time interval during which path $\alpha$ is perceived to be the cheaper path (in red, between $t_{1}^{+}$and $t_{2}^{+}$).

\footnotetext{
1 An analogous equation holds for $f_{\beta}$.

2 In the two-path system, $f_{\alpha} \geq \frac{1}{2}$ is equivalent to $c_{\alpha} \geq c_{\beta}$.
} 
Clearly, the discrepancy between reality and perception of path costs is the source of oscillation, as the discrepancy leads to increasing load on a path even when it is no longer the cheaper path (i.e., between $t_{2}^{*}$ and $t_{2}^{+}$). Due to the periodicity of this phenomenon, there exists no limit $\Delta^{*}$ of load difference and the oscillation-prone system experiences oscillation. An interesting observation is that both amplitude $(A)$ and oscillation period $(2 W)$ increase with the staleness of the information ( $T$ ); any $T>0$ leads to oscillations, only $T=0$ ensures stability.

In contrast, if the strategy profile $v$ contains different path-selection strategies, an oscillation-prone system may experience stability (cf. example in Appendix A in the full-paper version [29]).

\subsection{Equilibria on path-selection strategies}

In general, Nash equilibria refer to strategy profiles that do not allow for beneficial selfish strategy changes by individual agents. In the context of path-selection strategies, a Nash equilibrium is thus given if every end-host cannot improve its utility by switching to an alternative path-selection strategy. More formally, a Nash equilibrium on path-selection strategies can be defined as follows:

Definition 5. A strategy profile $v^{*}$ is a Nash equilibrium on path-selection strategies (PSS equilibrium) in an oscillationprone system $O=\left(\Pi, r, p, T, A_{0}, v^{*}\right)$ if and only if all strategies $\sigma$ with $v^{*}(\sigma)>0$ have cost $C(\sigma \mid O)=C^{*}$ and all strategies $\sigma^{\prime}$ with $v^{*}\left(\sigma^{\prime}\right)=0$ have cost $C\left(\sigma^{\prime} \mid 0\right) \geq C^{*}$.

It remains to formally define the cost $C(\sigma \mid O)$ of a strategy $\sigma$ in an oscillation-prone system $O$ with global strategy profile $v$. First, we note that a global strategy profile $v$, together with an initial strategy-adoption distribution for each path, uniquely defines the flow dynamics $f(t)=\left(f_{\alpha}(t), f_{\beta}(t)\right)$ in oscillation-prone systems with two paths. As the flow share controlled by each agent is assumed to be negligible in the Wardrop model, the flow dynamics $f(t)$ are not affected by the choice of $\sigma$ when varying $\sigma$ for a single agent. The basic costs of the two path options $\alpha$ and $\beta$ at any moment $t$ are thus given by $c_{\alpha}(t)$ and $c_{\beta}(t)$, both uniquely defined by an oscillation-prone system $O=\left(\Pi, r, p, T, A_{0}, v\right)$.

Given expected re-evaluation periods of duration $R$, an end-host deciding for path $\pi$ at time $t$ incurs the usage cost

$$
c_{\mathrm{u}}(\pi, t)=\frac{1}{R} \int_{t}^{t+R} c_{\pi}(s) \text {. }
$$

At time $t$, the cost $c(\sigma, t)$ of applying a strategy $\sigma$ is

$$
c\left(\sigma, t \mid \pi^{\prime}\right)=\sum_{\pi \in \Pi} u\left(\pi, t \mid \pi^{\prime}\right) \cdot c_{\mathrm{u}}(\pi, t),
$$

where $\pi^{\prime}$ is the current path of the end-host before the decision at time $t$ and $u\left(\pi, t \mid \pi^{\prime}\right)$ is the probability that path $\pi$ is selected at time $t$ given the current path $\pi^{\prime}$.

Furthermore, the strategy also determines the probability distribution $y\left(\pi^{\prime} \mid t\right)$ that defines the probability of the current path being $\pi^{\prime}$ at time $t$. The expected cost for applying a strategy $\sigma$ at time $t$ is thus given as follows:

$$
C(\sigma, t)=\sum_{\pi^{\prime} \in \Pi} y\left(\pi^{\prime} \mid t\right) \cdot c\left(\sigma, t \mid \pi^{\prime}\right)
$$

The expected cost of applying a strategy $\sigma$ in general can be derived as the average time-dependent strategy cost during a certain relevant time span $\left[t_{0}, t_{1}\right]$ :

$$
C(\sigma \mid 0)=\frac{1}{t_{1}-t_{0}} \int_{t_{0}}^{t_{1}} C(\sigma, t) t
$$

For systems that converge to stability at equal load, the relevant time span extends from $t_{0}=0$ until time $t_{\delta}$ when the system has converged according to some criterion $\delta>0$, i.e., $\forall t>t_{\delta} . \Delta(t)<\delta$. The time after convergence does not have to be considered as all strategies have the same cost for a system with equal path costs. For periodic oscillating systems, the relevant time span is defined as every interval that contains the periodically repeated sub-function. For an example of a PSS equilibrium analysis, see Appendix B in the full-paper version [29].

\section{Limits of stable strategies}

In this section, we investigate whether the stability-inducing path-selection strategies proposed in the literature form PSS equilibria. The question is whether an end-host can minimize its cost with a stability-oriented strategy if that strategy is universally adopted.

We perform this investigation by means of two case studies. In Section 3.1, we analyze the convergent rerouting policies designed by Fischer and Vöcking [8] and show that such rerouting policies are not compatible with the selfishness of endhosts. In Section 3.2, we analyze the MATE algorithm [14] and show its equivalence to the rerouting policies discussed in Section 3.1. 


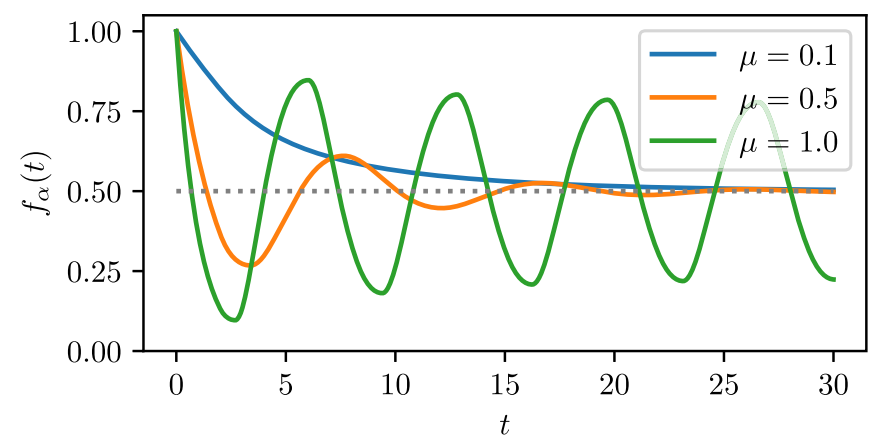

Fig. 2. Dynamics produced by universal adoption of strategy $\sigma_{\mathrm{c}}$ with different $\mu$ in oscillation-prone system $O=\left(\{\alpha, \beta\}, r=1, p=1, T=2, A_{0}=\right.$ $\left.1, v=\left\{\sigma_{\mathrm{c}} \mapsto 1\right\}\right)$.

\subsection{Rerouting Policies by Fischer E Vöcking}

A typical example of a convergent path-selection strategy has been proposed by Fischer and Vöcking [8]. The proposed path-selection strategy, which we henceforth refer to as the convergent strategy $\sigma_{\mathrm{c}}$, works as follows: If an end-host discovers a path with lower cost according to stale information, the end-host switches to that path with a probability that is a linear function of the perceived latency difference. More formally, the probability $u(\pi, t \mid \tilde{\pi})$ to switch from path $\pi_{t}$ to path $\pi \neq \tilde{\pi}$ at time $t$ is:

$$
u(\pi, t \mid \tilde{\pi})= \begin{cases}\mu \cdot \frac{c_{\tilde{\pi}}(t-T)-c_{\pi}(t-T)}{\Delta_{\max }} & \text { if } c_{\pi}(t-T)<c_{\tilde{\pi}}(t-T), \\ 0 & \text { otherwise, }\end{cases}
$$

Here, $\mu$ is a parameter in $[0,1]$ and the latency difference is normalized by $\Delta_{\max }$, which is 1 in parallel-path systems as defined in Section 2.1. The dynamics of a two-path oscillation-prone system where strategy $\sigma_{\mathrm{c}}$ is universally adopted can thus be described by the delay-differential equation (DDE)

$$
\frac{\partial f_{\alpha}}{\partial t}= \begin{cases}r \cdot \mu \cdot \Delta c(t-T) \cdot f_{\alpha}(t) & \text { if } \Delta c(t-T) \leq 0, \\ r \cdot \mu \cdot \Delta c(t-T) \cdot f_{\beta}(t) & \text { otherwise, }\end{cases}
$$

where $\Delta c(t-T)=c_{\beta}(t-T)-c_{\alpha}(t-T)$. This DDE describes a damped oscillator with delayed feedback and does not have an explicit solution [30]. However, we can numerically compute a solution using the method of steps [31].

As Fig. 2 shows, the choice of the parameter $\mu$ is critical for the strategy to actually lead to convergence. For high values of $\mu$, such as 1 , the strategy fails to produce convergence and yields undamped periodic oscillations. For low values of $\mu$, such as 0.1 , the system monotonically approaches the equilibrium without overshooting, i.e., it is overdamped (or, if nearly avoiding overshooting, critically damped). For values in-between, such as 0.5 , the system eventually converges to stability at equal load, but only after overshooting, i.e., it is underdamped. However, for both the overdamped and the underdamped convergent strategies, we can make the following observation:

Observation 1. Universal adoption of the convergent path-selection strategy $\sigma_{\mathrm{c}}$ does not represent a PSS equilibrium, neither in its underdamped nor in its overdamped variant.

In the case of the overdamped strategy (e.g., $\sigma_{\mathrm{c}}$ with $\mu=0.1$ ), the link loads monotonically approach each other and thus the greedy strategy allows an end-host to make use of a cheaper path sooner, making it the best-response strategy given universal adoption of $\sigma_{\mathrm{c}}$. In the case of the underdamped convergent strategy (e.g., $\sigma_{\mathrm{c}}$ with $\mu=0.5$ ), the fact that the strategy is not a PSS equilibrium in general is not obvious. However, we can show that there exist alternative strategies to the underdamped rerouting policy that reduce a deviant agent's cost, see Appendix C in the full-paper version [29].

\subsection{MATE algorithm}

The MATE algorithm [14] was designed for the intra-domain context, where an ingress router has to distribute its demand $d$ between multiple label-switched paths to a given egress router. As these ingress routers are under control of the domain operator, the MATE algorithm pursues convergence to the socially optimal traffic distribution, which minimizes latency from a global perspective, but is generally unstable given selfish end-hosts. In the context of inter-domain networks, the MATE algorithm is instantiated such that it converges to a Wardrop equilibrium, a type of equilibrium that is stable under the assumption of selfish agents.

We analyze whether applying the MATE algorithm is rational from an end-host's perspective. An end-host in an oscillation-prone two-path system would execute the MATE algorithm as follows. In every re-evaluation, the end-host 
selfishly optimizes its traffic allocation $\left(F_{\alpha}, F_{\beta}\right)^{\top}$, where $F_{\alpha}=d-F_{\beta}$. In order to conform to the Wardrop model, the demand $d$ is negligible from a global perspective. A MATE optimization step is defined as follows:

$$
\left(\begin{array}{l}
F_{\alpha}^{\prime} \\
F_{\beta}^{\prime}
\end{array}\right)=\left[\left(\begin{array}{l}
F_{\alpha} \\
F_{\beta}
\end{array}\right)-\gamma \cdot\left(\begin{array}{c}
c_{\alpha}(t-T) \\
c_{\beta}(t-T)
\end{array}\right)\right]^{+}
$$

In order to reach convergence despite stale information, the coefficient $\gamma$ has to conform to a certain upper bound [14]. Moreover, $[\mathbf{F}]^{+}$represents a projection of allocation vector $\mathbf{F}$ to the feasible allocation set defined by $F_{\alpha}+F_{\beta}=d$ with $F_{\alpha}, F_{\beta} \geq 0$.

As we show in Appendix D in the full-paper version [29], the dynamics of an oscillation-prone system with universal adoption of the MATE algorithm are described by the following differential equation:

$$
\frac{\partial f_{\alpha}}{\partial t}= \begin{cases}r \cdot \frac{\gamma}{2} \cdot \Delta(t-T) \cdot f_{\alpha}(t) & \text { if } \Delta(t-T) \leq 0 \\ r \cdot \frac{\gamma}{2} \cdot \Delta(t-T) \cdot f_{\beta}(t) & \text { otherwise }\end{cases}
$$

This equation is clearly equivalent to Eq. (12) for a choice of $\mu=\gamma / 2$. An oscillation-prone system with universal adoption of $\sigma_{\mathrm{c}}$ and a system with universal adoption of the MATE algorithm thus exhibit the same flow dynamics, which allow for beneficial deviation:

Observation 2. The path-selection strategy as prescribed by the MATE algorithm is equivalent to the path-selection strategy $\sigma_{\mathrm{c}}$. Thus, universal adoption of the MATE algorithm neither constitutes a PSS equilibrium.

\subsection{Conclusion}

In summary, the kind of convergent path-selection strategies proposed in the literature cannot be assumed to be adopted by selfish end-hosts, as deviating from these strategies (e.g., by switching faster than prescribed by the strategy) is beneficial to an end-host.

Stability in a path-aware network architecture with selfish end-hosts can thus not be guaranteed by non-oscillatory path-selection strategies that prescribe a maximum rate of change to be respected by end-hosts. Instead, the network could employ mechanisms that incentivize end-hosts to follow non-oscillatory path-selection strategies. This finding reflects a similar result $[32,33]$ in the context of congestion control, namely that socially desirable behavior of end-hosts can only be enforced with network support.

\section{Stabilization mechanisms}

As argued in the previous section, rational end-hosts in networks with unrestricted path choice are unlikely to adopt convergent path-selection strategies. Therefore, there is a need for mechanisms that allow network operators to incentivize the adoption of path-selection strategies that induce stability at equal load, i.e., incentive-compatible stabilization mechanisms. First, we integrate the concept of traffic-steering mechanisms into our game-theoretic model (Section 4.1). Second, we specify in Section 4.2 the conditions under which these mechanisms are incentive-compatible.

\subsection{Traffic-steering mechanisms}

In order to affect the path-selection decisions of end-hosts in an oscillation-prone system $O$, a traffic-steering mechanism $\mathcal{M}$ needs to alter the strategy cost $C(\sigma \mid 0)$ for at least one path-selection strategy $\sigma$. A mechanism $\mathcal{M}$ thus defines a function $c_{\mathcal{M}}(\pi, t)$ that quantifies the mechanism-imposed cost for using path $\pi$ at time $t$. This cost is imposed onto the user of a path $\pi$ in addition to the load-dependent path cost.

If a mechanism $\mathcal{M}$ is active, the usage $\operatorname{cost} c_{\mathrm{u}}^{\mathcal{M}}$ extends the standard usage $\operatorname{cost} c_{\mathrm{u}}$ from Eq. (7) as follows:

$$
c_{\mathrm{u}}^{\mathcal{M}}(\pi, t)=c_{\mathrm{u}}(\pi, t)+c_{\mathcal{M}}(\pi, t)
$$

The cost formulas $c^{\mathcal{M}}(\pi, t \mid \tilde{\pi}), C^{\mathcal{M}}(\sigma, t)$, and $C^{\mathcal{M}}(\sigma \mid 0)$ can be constructed from $c_{\mathrm{u}}^{\mathcal{M}}(\pi, t)$, analogously to Section 2.4.

\subsection{Incentive compatibility}

In general, incentive-compatible mechanisms are mechanisms that incentivize a certain form of desirable behavior. In our context, we consider traffic-steering mechanisms to be incentive-compatible if these mechanisms incentivize the desirable behavior of adopting a non-oscillatory path-selection strategy. In other words, an incentive-compatible mechanism creates a PSS equilibrium, i.e., a situation where every end-host minimizes its cost by adopting a non-oscillatory path-selection strategy, given that all other end-hosts do so:

Definition 6. A traffic-steering mechanism $\mathcal{M}$ is an incentive-compatible stabilization mechanism for an oscillationprone system $O$ if there is a strategy profile $v^{*}$ such that 


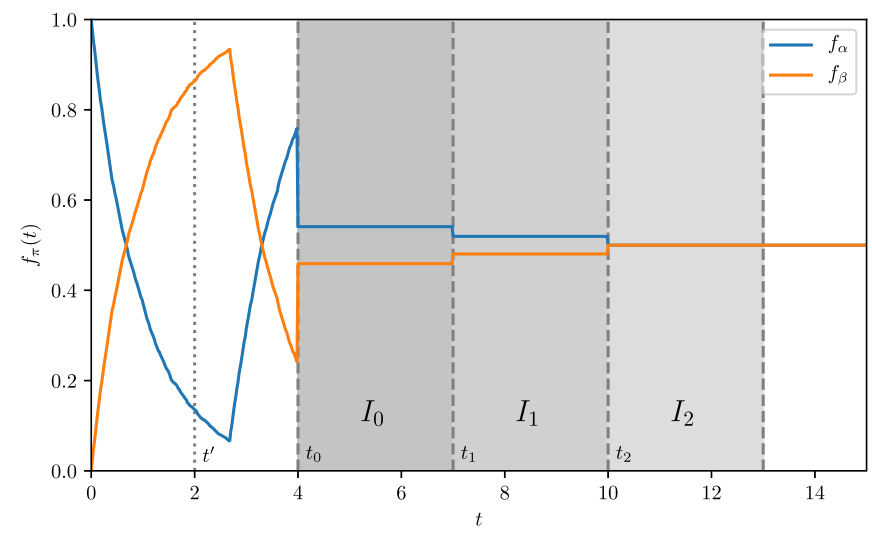

Fig. 3. Simulation of FLOSS enforcement in an oscillation-prone system $O=\left(\Pi=\{\alpha, \beta\}, r=1, p=1, T=2, A_{0}=1, v=\left\{\sigma_{\mathrm{F}} \mapsto 1\right\}\right)$.

(i) $v^{*}$ leads to stability at equal load and

(ii) $v^{*}$ represents a PSS equilibrium with respect to the cost function $C^{\mathcal{M}}(\sigma \mid 0)$.

In the following two sections, we present two instances of stabilization mechanisms, namely FLOSS and CROSS, and prove their incentive compatibility. The two mechanisms differ in the methods for achieving stability: Whereas FLOSS reduces the imbalance between two paths by regulating the migration rate between the paths, CROSS achieves stability by repetitive reshuffling of flows between paths and increasing the cost of path migration.

\section{The FLOSS mechanism}

In this section, we present the FLOSS mechanism (Flow-Loyalty Oscillation-Suppression System).

\subsection{Overview}

As shown in Section 3, convergent path-selection strategies are characterized by careful path-switching behavior: An end-host only switches to a seemingly cheaper path with a modest probability that depends on the measured latency difference, translating into a relatively low migration rate between paths. It is well known that system stability can be achieved by limiting the rate of change (also known as the system gain [17]). However, the challenge is to develop methods that achieve this change-rate limitation in the face of selfish, uncontrolled end-hosts. Such a method is given by FLOSS.

As selfish end-hosts do not voluntarily conform to a modest path-migration rate, the path-migration rate has to be regulated by network operators. The FLOSS mechanism performs such regulation by rewarding end-hosts that are loyal to a certain path and by restricting arbitrary path migration by oscillating end-hosts.

In order to regulate path migration, the FLOSS mechanism makes use of registrations and proceeds in intervals. Fig. 3 , which shows a simulation of the FLOSS mechanism in a two-path system, illustrates the FLOSS approach. Initially, the FLOSS mechanism announces at time $t^{\prime}$ that all end-hosts are required to obtain a registration for one path $\pi$ of their choice. This registration allows an end-host to use path $\pi$ during a future time interval $I_{0}=\left[t_{0}, t_{1}\right)$ with $t^{\prime}<t_{0}<t_{0}+T<$ $t_{1}$. End-hosts that use path $\pi$ without a registration are punished in the interval (e.g., by dropping packets).

This call for registration produces a distribution of flows over the two paths, which is stable during the interval as no end-host can switch to the path which it is not registered for. However, this load distribution is unlikely to be perfectly equal. The FLOSS mechanism iteratively reduces this imbalance: In every following time interval, a small set of flows are allowed to migrate from the more expensive path to the cheaper path. This allowance is enforced by selectively granting registrations: Whereas end-hosts with a pre-existing registration for a path (loyal end-hosts) always obtain a registration for that path, end-hosts without a pre-existing registration are not always allowed to register. Once the imbalance is sufficiently small, the end-hosts do not have an incentive anymore to switch paths, at which point the enforcement of the mechanism can be suspended (e.g., at the end of interval $I_{2}$ in Fig. 3).

Theorem 1. The FLOSS mechanism is an incentive-compatible stabilization mechanism.

As defined in Section 4.2, incentive compatibility implies the existence of a strategy profile that leads to stability at equal load and is a PSS equilibrium during mechanism enforcement. For FLOSS, such a strategy profile is given by universal adoption of the FLOSS-compliant path-selection strategy $\sigma_{\mathrm{F}}$. The strategy $\sigma_{\mathrm{F}}$ prescribes to use the path with the lowest expected cost which the end-host is entitled to use. Our incentive-compatibility proof thus builds on the following two concrete lemmas, which are proved in Sections 5.2 and 5.3, respectively: 
Lemma 1. Universal adoption of the FLOSS path-selection strategy $\sigma_{\mathrm{F}}$ leads to stability at equal load.

Lemma 2. Universal adoption of the FLOSS path-selection strategy $\sigma_{\mathrm{F}}$ represents a PSS equilibrium during enforcement of the FLOSS mechanism.

\subsection{Stability analysis}

In order to prove Lemma 1, we assume universal adoption of path-selection strategy $\sigma_{\mathrm{F}}$, i.e., an end-host always uses the path with the lower expected cost provided that the end-host is entitled to use that path.

When registering before the initial interval, all end-hosts simultaneously decide for one path to use during the upcoming interval $\left[t_{0}, t_{1}\right)$. Confronted with such a choice, each end-host aspires to commit to the path $\pi$ that will be selected by fewer other end-hosts, i.e., the path $\pi$ with $f_{\pi}\left(t_{0}\right)<f_{\tilde{\pi}}\left(t_{0}\right)$. In absence of inherent differences between the two choices, the only Nash equilibrium of such a speculative game is given if every end-host commits to each path $\pi$ with probability $1 / 2$.

In expectation, the load on both paths $\alpha$ and $\beta$ is thus $\mathbb{E}\left[f_{\alpha}\left(t_{0}\right)\right]=\mathbb{E}\left[f_{\beta}\left(t_{0}\right)\right]=1 / 2$. Since no migration occurs during the interval $\left[t_{0}, t_{1}\right)$, the load distribution is expected to remain equal during the interval, i.e., $\mathbb{E}\left[f_{\alpha}(t)\right]=\mathbb{E}\left[f_{\beta}(t)\right]=1 / 2$ $\forall t \in\left[t_{0}, t_{1}\right)$. When mechanism enforcement ends at time $t_{1}$, the end-hosts are again free to arbitrarily select paths. However, since $t_{0}+T<t_{1}$, any end-host performing a re-evaluation after $t_{1}$ perceives the Wardrop equilibrium $c_{\alpha}(t-T)=c_{\beta}(t-T)$ and will thus not switch paths. Therefore, the system is stable at equal load even when the mechanism is not enforced anymore.

In reality, however, variance makes it likely that the load on paths $\alpha$ and $\beta$ is not perfectly equalized at $t_{0}$. In that case, the FLOSS mechanism attempts to eliminate the remaining load difference $\Delta\left(t_{0}\right)=\left|f_{\alpha}\left(t_{0}\right)-f_{\beta}\left(t_{0}\right)\right|>0$ as follows. Starting from $t^{\prime \prime}=t_{0}+T$, the end-hosts can again register on paths for an upcoming interval [ $\left.t_{1}, t_{2}\right)$. At $t^{\prime \prime}$, all end-hosts correctly perceive the cost difference between a cheaper path $\pi$ and a more expensive path $\tilde{\pi}$, as for every path $\hat{\pi}$, $c_{\hat{\pi}}\left(t^{\prime \prime}-T\right)=c_{\hat{\pi}}\left(t_{0}\right)=c_{\hat{\pi}}\left(t^{\prime \prime}\right)$ due to the constant load in $\left[t_{0}, t^{\prime \prime}\right)$. The core idea of the FLOSS mechanism is to determine and enforce a migration allowance $\rho_{\pi}\left(t_{1}\right)$, which is an upper bound on the amount of end-hosts that are allowed migrate from path $\tilde{\pi}$ to path $\pi$ at time $t_{1}$.

Importantly, $\rho_{\pi}\left(t_{1}\right)$ is chosen such that

$$
f_{\pi}\left(t_{0}\right)+\rho_{\pi}\left(t_{1}\right) \cdot f_{\tilde{\pi}}\left(t_{0}\right) \leq\left(1-\rho_{\pi}\left(t_{1}\right)\right) \cdot f_{\tilde{\pi}}\left(t_{0}\right),
$$

which implies $c_{\pi}\left(t_{1}\right) \leq c_{\tilde{\pi}}\left(t_{1}\right)$ (i.e., the cheaper path $\pi$ will remain the cheaper path in the next interval even if a share $\rho_{\pi}\left(t_{1}\right)$ of end-hosts on the more expensive path $\tilde{\pi}$ migrate to path $\left.\pi\right)$. This choice of $\rho_{\pi}\left(t_{1}\right)$ ensures the correct incentives for the end-hosts. Given such an assurance, end-hosts registered on the cheaper path $\pi$ during $\left[t_{0}, t_{1}\right)$ minimize their cost by remaining on path $\pi$. Since these end-hosts are considered loyal to path $\pi$, their registration at path $\pi$ will be renewed for the upcoming interval $\left[t_{1}, t_{2}\right)$. Conversely, all end-hosts registered on the more expensive path $\tilde{\pi}$ would minimize their cost by migrating to the cheaper path $\pi$. However, the FLOSS mechanism restricts this migration by only granting a registration for $\pi$ to a share $\rho_{\pi}\left(t_{1}\right)$ of end-hosts on $\tilde{\pi}$. The non-migrating end-hosts on path $\tilde{\pi}$ are considered loyal on path $\tilde{\pi}$ and are thus allowed to renew their registration at $\tilde{\pi}$.

Therefore, exactly $\rho_{\pi}\left(t_{1}\right) \cdot f_{\tilde{\pi}}\left(t_{0}\right)$ migrate from path $\tilde{\pi}$ to path $\pi$ at time $t_{1}$, which reduces the difference in load and cost between the paths $\pi$ and $\tilde{\pi}$. By repetitive mechanism application with appropriately chosen migration allowances, the FLOSS mechanism can arbitrarily minimize the cost differential between the paths $\pi$ and $\tilde{\pi}$. When the cost difference becomes so small that end-hosts perceive a Wardrop equilibrium, the mechanism has achieved stability at equal load that continues to hold even without mechanism enforcement.

\subsection{PSS equilibrium analysis}

We now prove Lemma 2, i.e., we show that path-selection strategy $\sigma_{\mathrm{F}}$ is the optimal strategy for an end-host given that all other end-hosts have adopted $\sigma_{\mathrm{F}}$. Concretely, we show that the FLOSS mechanism induces a PSS equilibrium $v^{*}=\left\{\sigma_{\mathrm{F}} \mapsto 1\right\}$, where $\sigma_{\mathrm{F}}$ is the universally adopted path-selection strategy with the following path-selection function:

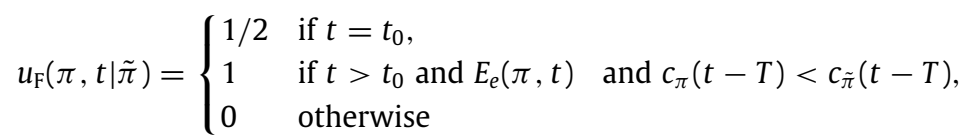

where $E_{e}(\pi, t)$ is true if and only if end-host $e$ is entitled to use path $\pi$ at time $t$. We assume that an end-host always knows whether it is entitled to use a path. For the initial interval, every path is selected with equal probability $1 / 2$. For all subsequent intervals, a path $\pi$ is selected if the path is perceived to be cheaper than the current path $\tilde{\pi}$ and end-host $e$ is entitled to use path $\pi$. For remaining on a path $\tilde{\pi}$, it holds that $u_{\mathrm{F}}(\tilde{\pi}, t \mid \tilde{\pi})=1-u_{\mathrm{F}}(\pi, t \mid \tilde{\pi})$.

The FLOSS mechanism makes strategy $\sigma_{\mathrm{F}}$ the equilibrium strategy by imposing the additional cost $c_{\mathcal{M}}(\pi, t)$ for using path $\pi$ at time $t$. End-host $e$ incurs a cost $c_{\mathrm{a}}$ for attempting to register and a penalty cost $c_{\mathrm{p}}$ for using a path without a registration. We assume $c_{\mathrm{p}}=\infty$, i.e., the penalty cost makes a path unusable. Let $A_{e}(\pi, t)$ be true if and only if end-host $e$ 
applies to register for using path $\pi$ at time $t$ and let $R_{e}(\pi, t)$ be true if and only if end-host $e$ obtained a registration for using path $\pi$ at time $t$, i.e., $R_{e}(\pi, t)=A_{e}(\pi, t) \wedge E_{e}(\pi, t)$. Using these predicates, the cost imposed by the FLOSS mechanism can be expressed as

$$
c_{\mathcal{M}}\left(\pi, t \mid A_{e}, R_{e}\right)=\left[A_{e}(\pi, t)\right] \cdot c_{\mathrm{a}}+\left[\neg R_{e}(\pi, t)\right] \cdot c_{\mathrm{p}},
$$

where $[P]=1$ if the predicate $P$ is true and 0 otherwise.

A selfish end-host $e$ chooses its actions such that its cost from the mechanism is minimized. Therefore, an end-host $e$ requests a registration if and only if the end-host is entitled to the registration, as there is no benefit of a registration request that will be refused. Thus the relevant mechanism-imposed cost for end-host $e$ is

$$
c_{\mathcal{M}}(\pi, t)=\min _{A_{e}, R_{e}} c_{\mathcal{M}}\left(\pi, t \mid A_{e}, R_{e}\right)= \begin{cases}c_{\mathrm{a}} & \text { if } E_{e}(\pi, t), \\ c_{\mathrm{p}} & \text { otherwise. }\end{cases}
$$

Concerning the initial interval with start $t_{0}$, both paths $\alpha$ and $\beta$ have expected cost $c_{\pi}\left(t_{0}\right)=1 / 2^{p}$ if all other endhosts choose each path with probability $u_{\mathrm{F}}(\pi, t \mid \tilde{\pi})=1 / 2$. As both paths have the same cost and both paths require a registration, the usage cost of both paths is $c_{\mathrm{u}}^{\mathcal{M}}\left(\pi, t_{0}\right)=1 / 2^{p}+c_{\mathrm{a}}$. Independent of the current path $\tilde{\pi}$, the cost of applying strategy $\sigma_{\mathrm{F}}$ at time $t_{0}$ is thus $c^{\mathcal{M}}\left(\sigma_{\mathrm{F}}, t_{0} \mid \tilde{\pi}\right)=1 / 2^{p}+c_{\mathrm{a}}$ for any choice of $u\left(\pi, t_{0} \mid \tilde{\pi}\right)$. Therefore, end-host $e$ cannot reduce its cost by choosing another path-selection probability than $u_{\mathrm{F}}\left(\pi, t_{0} \mid \tilde{\pi}\right)=1 / 2$, which makes $\sigma_{\mathrm{F}}$ an equilibrium strategy for the initial interval.

Concerning subsequent intervals with start $t_{i}>t_{0}$, we have to distinguish two cases for the current path $\pi^{\prime}$ of end-host $e$, namely whether end-host $e$ is on the cheaper path $\pi$ or on the more expensive path $\tilde{\pi}^{3}$

1. If end-host $e$ is on the cheaper path $\pi$, the cost of remaining on $\pi$ is $c_{u}^{\mathcal{M}}\left(\pi, t_{i}\right)=c_{\pi}\left(t_{i}\right)+c_{\mathrm{a}}$, whereas the cost of switching to $\tilde{\pi}$ is $c_{\mathrm{u}}^{\mathcal{M}}\left(\tilde{\pi}, t_{i}\right)=c_{\tilde{\pi}}\left(t_{i}\right)+c_{\mathrm{a}}$ if $E_{e}\left(\tilde{\pi}, t_{i}\right)$ and $c_{\tilde{\pi}}\left(t_{i}\right)+c_{\mathrm{p}}$ otherwise. As always $c_{\mathrm{u}}^{\mathcal{M}}\left(\pi, t_{i}\right)<c_{\mathrm{u}}^{\mathcal{M}}\left(\tilde{\pi}, t_{i}\right)$, the current path $\pi$ must be selected with probability $u(\pi, t \mid \pi)=1$ to minimize the end-host's cost.

2. If end-host $e$ is on the more expensive path $\tilde{\pi}$, the cost of remaining on $\tilde{\pi}$ is $c_{\mathrm{u}}^{\mathcal{M}}\left(\tilde{\pi}, t_{i}\right)=c_{\tilde{\pi}}\left(t_{i}\right)+c_{\mathrm{a}}$, whereas the cost of switching to $\pi$ is $c_{\mathrm{u}}^{\mathcal{M}}\left(\pi, t_{i}\right)=c_{\pi}\left(t_{i}\right)+c_{\mathrm{a}}$ if $E_{e}\left(\pi, t_{i}\right)$ and $c_{\pi}\left(t_{i}\right)+c_{\mathrm{p}}$ otherwise. Thus, $c_{\mathrm{u}}^{\mathcal{M}}\left(\pi, t_{i}\right)<c_{\mathrm{u}}^{\mathcal{M}}\left(\tilde{\pi}, t_{i}\right)$ if $E_{e}\left(\pi, t_{i}\right)$, but $c_{\mathrm{u}}^{\mathcal{M}}\left(\tilde{\pi}, t_{i}\right)<c_{\mathrm{u}}^{\mathcal{M}}\left(\pi, t_{i}\right)$ otherwise. If end-host $e$ is entitled to use the cheaper path $\pi$, the cheaper path $\pi$ must thus be selected with probability $u(\pi, t \mid \tilde{\pi})=1$ to minimize the end-host's cost, and with probability 0 otherwise.

In summary, for all intervals with start $t_{i}>t_{0}$, an end-host $e$ optimizes its cost by switching to an alternative path $\pi$ if and only if path $\pi$ is cheaper than the current path $\tilde{\pi}$ and end-host $e$ is entitled to use path $\pi$. This path-switching behavior is exactly captured by the path-selection function $u_{\mathrm{F}}(\pi, t \mid \tilde{\pi})$. Therefore, path-selection strategy $\sigma_{\mathrm{F}}$ is an equilibrium strategy for both the initial interval and the subsequent intervals of the mechanism, which proves Lemma 2.

\section{The CROSS mechanism}

In this section, we present a second stabilization mechanism called CROSS (Computation-Requiring Oscillation Suppression System).

\subsection{Overview}

While the FLOSS mechanism (cf. Section 5) deterministically achieves stability at equal load, its strict enforcement of the migration allowance represents a problem in case of path failures. When a path fails, an end-host on that path is not allowed to switch to an alternative path immediately. Only when the path failure is detected after some time by the mechanism, enforcement of the mechanism can be stopped and the end-hosts can be allowed to use an alternative path. For highly critical transmissions, such inflexibility is undesirable.

The CROSS mechanism allows end-hosts to obtain an insurance against such cases of path failure. Basically, the CROSS mechanism works similarly to the initial interval of the FLOSS mechanism: End-hosts are required to register for one path of their choice, which in general cannot be changed during the upcoming interval. Unlike FLOSS, however, the CROSS mechanism offers the possibility of registration for a second path that can be immediately used in case of a path failure, even if the path failure is not yet verified.

However, the question is how to avoid that end-hosts always register for both paths and, if on the more expensive path, falsely claim to be affected by a path failure and switch to the cheaper path. Such opportunistic behavior would cause oscillation. To solve this problem, the idea of the CROSS mechanism is that end-hosts must prove that they need the immediate-switching option for insurance against path failures, not simply for opportunistic cost reduction. End-hosts can prove their truthfulness by paying a price for the immediate-switch option. This price must be higher than any cost gain that can be achieved by switching to a cheaper path in a scenario without path failure. An end-host that paid this

\footnotetext{
3 Thanks to the load being constant in subsequent intervals, the cost $c_{\hat{\pi}}(t)$ of a path $\hat{\pi}$ at registration time $t$ is equal to the known stale cost $c_{\hat{\pi}}(t-T)$. Therefore, any end-host can correctly identify the cheaper and the more expensive path.
} 


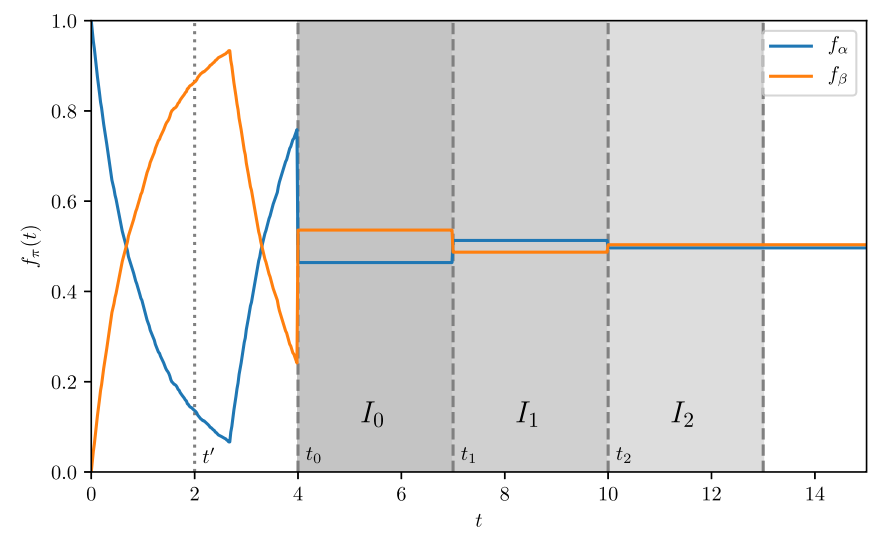

Fig. 4. Simulation of CROSS enforcement in an oscillation-prone system $O=\left(\Pi=\{\alpha, \beta\}, r=1, p=1, T=2, A_{0}=1, v=\left\{\sigma_{\mathrm{C}} \mapsto 1\right\}\right)$, with $\epsilon=0.01$.

price thus only switches to the backup path if a path failure has occurred; if no path failure occurred, the end-host would not trade its insurance option against the cost gain, as the insurance option is more valuable to the end-host than any cost gain. Immediate switching during the interval can thus be allowed to the end-hosts with a backup-path registration. Moreover, immediate switching behavior by those end-hosts is an indication of path failure, which means that all other end-hosts must be allowed to migrate as well.

As a price for the backup path registration, the CROSS mechanism requires the solution to a computationally hard puzzle. This puzzle is structured such that only end-hosts with a sufficiently high valuation of the backup path will obtain a solution. More precisely, each puzzle $\mathcal{E}$ is associated with a cryptographic hash function $h:\{0,1\}^{*} \mapsto[0,1]$ and a difficulty level $\delta \geq 0$. An end-host $e$ can solve a puzzle $\mathcal{E}(\pi)$ for registering at a backup path $\pi$ by finding a value $s$ such that $h\left(\pi, t_{i}, e, s\right) \leq 2^{-\delta}$, where $t_{i}$ is the start of the next balancing trial. Given a cryptographic hash function, a puzzle $\mathcal{E}(\pi)$ can only be solved by brute force, i.e., varying $s$ in a series of hash computations. By finding an appropriate $s$, an end-host can obtain a backup-path registration.

Also unlike FLOSS, the CROSS mechanism allows end-hosts to register at a path of their choice not only for the initial interval, but for every interval. Therefore, even if the path failure is not detected for some reason (e.g., because no end-host obtained a backup registration), the end-host can use the alternative path in the interval after a path failure. The CROSS mechanism thus has a non-deterministic approach for achieving stability: Intervals in CROSS serve as balancing trials and are repeated until the load imbalance is small enough that end-hosts do not switch paths anymore. Since the end-hosts select each path with probability $1 / 2$ in any balancing trial, the probability that an approximately equal load distribution results after a few balancing trials is substantial. Still, the additional flexibility of CROSS results in a loss of convergence guarantees: Instead of convergence to an equal-load distribution, the CROSS mechanism only guarantees convergence to a traffic distribution with approximately equal load. A simulation of CROSS enforcement is visualized in Fig. 4, which also shows the convergence produced by the CROSS approach.

Theorem 2. The CROSS mechanism is an incentive-compatible stabilization mechanism that achieves stability at approximately equal load, i.e., for every $\epsilon>0, \lim _{t \rightarrow \infty} \Delta(t)<\epsilon$.

The CROSS mechanism achieves stability at approximately equal load by incentivizing the universal adoption of pathselection strategy $\sigma_{\mathrm{C}}$, which prescribes that end-hosts only use a path if they have a corresponding registration and only use a backup in case of path failures. More formally, Theorem 2 directly follows from Lemmas 3 and 4 :

Lemma 3. Universal adoption of the CROSS path-selection strategy $\sigma_{\mathrm{C}}$ leads to stability at approximately equal load.

Lemma 4. Universal adoption of the CROSS path-selection strategy $\sigma_{\mathrm{C}}$ represents a PSS equilibrium given enforcement of the CROSS mechanism.

While the proof of Lemma 3 is intuitive and can thus be found in Appendix E of the full-paper version [29], Lemma 4 is proven below. 


\subsection{PSS equilibrium analysis}

In this section, we prove Lemma 4 by showing that universal adoption of path-selection strategy $\sigma_{\mathrm{C}}$ is a PSS equilibrium, i.e., if all other end-hosts adopt $\sigma_{\mathrm{C}}, \sigma_{\mathrm{C}}$ is the optimal strategy for a single end-host $e$. The path-selection strategy $\sigma_{\mathrm{C}}$ is characterized by the following path-selection function for $\pi \neq \tilde{\pi}$ :

$$
u_{\mathrm{C}}(\pi, t \mid \tilde{\pi})= \begin{cases}1 / 2 & \text { if } t=t_{i} \wedge \neg R_{e}^{\prime}(\pi, t), \\ 1 & \text { if } c_{\tilde{\pi}}(t-T)=\infty \wedge R_{e}^{\prime}(\pi, t), \\ 0 & \text { otherwise, }\end{cases}
$$

where $t_{i}$ is the start time of any balancing trial, $c_{\tilde{\pi}}(t-T)=\infty$ designates a path failure and $R_{e}^{\prime}(\pi, t)$ is true if and only if end-host $e$ has a backup registration for path $\pi$ at time $t$. Moreover, $u_{\mathrm{C}}(\tilde{\pi}, t \mid \tilde{\pi})=1-u_{\mathrm{C}}(\pi, t \mid \tilde{\pi})$.

As in FLOSS, registering has cost $c_{\mathrm{a}}$, whereas using a path without registration imposes a penalty cost $c_{\mathrm{p}}=\infty$. Additionally, an end-host incurs cost by solving puzzles, where each hashing operation has cost $c_{\mathrm{h}}$. To an end-host with valuation $\omega$ of a backup path, a hash operation has the expected utility $\mathbb{E}\left[U_{\mathrm{h}}\right](\delta, \omega)=2^{-\delta} \omega-c_{\mathrm{h}}$.

Given puzzle-difficulty level $\delta$, an end-host thus solves a puzzle if and only if it has a backup valuation $\omega$ such that $\mathbb{E}\left[U_{\mathrm{h}}\right](\delta, \omega)>0$. If an end-host does not solve a puzzle, it simply obtains a regular registration for one path at cost $c_{\mathrm{a}}$, where every path is selected with probability $1 / 2$. Obtaining no registration and using any path would incur a much higher penalty $\operatorname{cost} c_{\mathrm{p}} \gg c_{\mathrm{a}}$ and is thus not rational. Therefore, an end-host with a registration for one path uses this path from the start $t_{i}$ of the balancing trial. If an end-host solves a puzzle, the end-host obtains a backup registration for the path corresponding to the puzzle and obtains a regular registration for the other path at cost $c_{\mathrm{a}}$. Since CROSS enforces that an end-host can only switch once to its backup path and never switch back during the balancing trial, every end-host with a backup-path registration starts by using the path with its regular registration at time $t_{i}$. In summary, the optimal path-selection function for all $t=t_{i}$ is $u_{\mathrm{C}}\left(\pi, t \mid \pi^{\prime}\right)=1 / 2$ if $\neg R_{e}^{\prime}(\pi, t)$.

During the balancing trial, no reallocation decisions are taken before $t_{i}+T$, as the expected path costs during $\left[t_{i}, t_{i}+T\right]$ is $\mathbb{E}\left[c_{\alpha}\right]=\mathbb{E}\left[c_{\beta}\right]=1 / 2^{p}$. Only at $t_{i}+T$, the actual imbalance $\Delta(t)=\left|f_{\pi}\left(t_{i}\right)-f_{\tilde{\pi}}\left(t_{i}\right)\right|$ between a more expensive path $\tilde{\pi}$ and a cheaper path $\pi$ becomes visible to the end-hosts. If the end-hosts on path $\tilde{\pi}$ with a backup registration for path $\pi$ switched at that point, they would save $\Delta \bar{C}=\int_{t_{i}+T}^{t_{i+1}}\left(c_{\tilde{\pi}}(t)-c_{\pi}(t)\right) \mathrm{d} t$, which is bounded above by $\Delta \bar{C}_{\max }=t_{i+1}-t_{i}-T$. However, such a switch would erase the backup value $\omega$ of path $\pi$ for the end-host, which is why an end-host with backup registration for path $\pi$ only switches to path $\pi$ if $\omega<\Delta \bar{C}$. In order to disincentivize such migration and keep the load distribution constant, the CROSS mechanism chooses the puzzle-difficulty level $\delta$ such that $\mathbb{E}\left[U_{\mathrm{h}}\right](\delta, v)>0$ if and only if $\omega>\Delta \bar{C}_{\max }$. This choice of $\delta$ leads to a situation where the end-hosts with a backup registration will only switch to the backup path in case of a path failure, as these end-hosts value the backup option higher than any cost reduction obtainable without path failure. In case of a path failure, however, trading the backup value $\omega$ of path $\pi$ against the infinite cost of failed path $\tilde{\pi}$ is rational and the end-hosts with a backup registration switch the paths. In summary, the optimal path-selection function for end-host $e$ and for all $t \neq t_{i}$ is thus $u_{\mathrm{C}}(\pi, t \mid \tilde{\pi})=1$ if $R_{e}^{\prime}(\pi, t)$ and $c_{\tilde{\pi}}(t-T)=\infty$, and $u_{C}(\pi, t \mid \tilde{\pi})=0$ otherwise. Thereby, path-selection strategy $\sigma_{C}$ has been established as the PSS equilibrium strategy.

\section{Practical application}

While the focus of this paper is on the theoretical exploration of selfish path selection and stabilization mechanisms, this section lays out a pathway towards practical application of our findings. First, we discuss practical requirements for inter-domain stabilization mechanisms in Section 7.1. In Section 7.2, we present a mechanism-enforcement architecture that conforms to these requirements. In Sections 7.3 and 7.4, we outline how the FLOSS and CROSS mechanisms could be practically implemented.

\subsection{Requirements}

If a stabilization mechanism is to be practically applied by network operators in an inter-domain architecture, the mechanism must conform to the following requirements:

1. Limited overhead: The stabilization mechanism must only induce a small overhead on the systems of network operators. In particular, the genuine function of AS border routers (forwarding traffic at line rate) must not be compromised by expensive mechanism-enforcement tasks. Note that both mechanisms only need to be enforced by routers in case of oscillation and until stabilization is achieved; however, the mechanisms should induce little overhead even during this short time span.

2. No explicit inter-AS coordination (coordination-freeness): The stabilization mechanism must not rely on explicit interAS coordination. Such explicit coordination may not be feasible or scalable, as the domains that perceive the same oscillation pattern may be mutually unknown, mutually distrusted, or very distant from each other. 


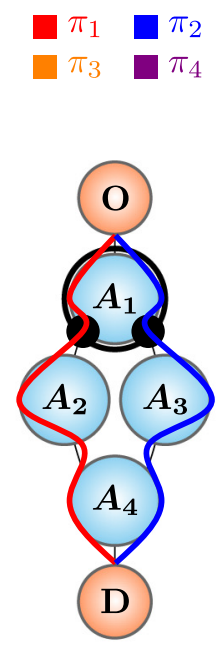

(a)

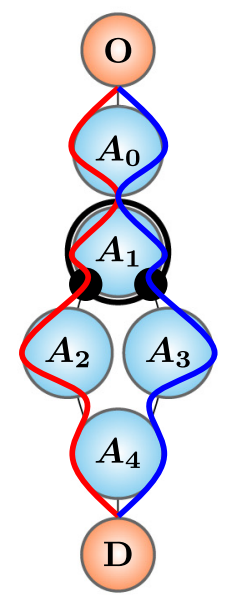

(b)

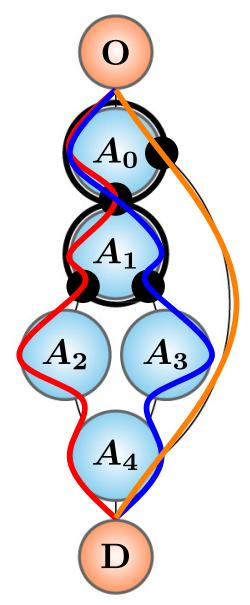

(c)

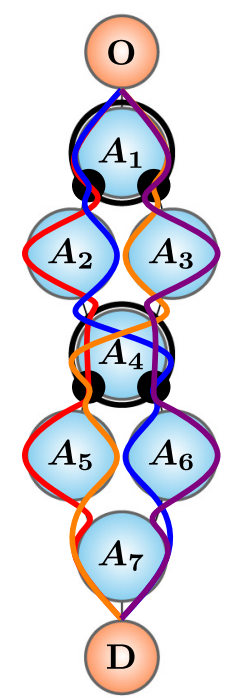

(d)

Fig. 5. Oscillation patterns.

\subsection{Mechanism-enforcement architecture}

To enforce a stabilization mechanism, an AS operator needs the means to detect, inform, and punish the selfish entities that employ an oscillatory path-selection strategy. In this section, we describe a mechanism-enforcement architecture that provides these means to an AS operator while conforming to the requirements in Section 7.1.

From an inter-domain perspective, the most important architectural question is the question of coordination, i.e., how each AS perceiving an oscillation pattern contributes to oscillation suppression. As explicit inter-AS coordination is undesirable, an implicit method for responsibility assignment is necessary.

We leverage a fundamental property of paths in inter-domain network graphs as a natural way to assign responsibility for inter-domain oscillation suppression. This fundamental property is based on the following insight: For every pair of paths connecting the same origin and destination ASes, there is at least one AS (henceforth: the splitting AS) in which the paths split, i.e., the paths contain different egress interfaces out of the AS. For every oscillation between two paths, there is thus at least one AS which perceives the oscillation as an oscillation of traffic between egress interfaces, not only as periodic upswings and downswings in the load at one egress interface. Such splitting ASes are the natural candidates for a leading role in inter-domain oscillation suppression, as these ASes are both best informed about the oscillation and in the best position to manage the oscillating traffic.

For illustration of the path-splitting property, Fig. 5 shows different types of oscillation patterns for paths connecting an origin end-host $O$ and a destination end-host $D$. In the simplest cases, the oscillation may be perceived at the origin AS (AS $A_{1}$ in Fig. 5(a)) or at one intermediate AS (AS $A_{1}$ in Fig. 5(b)). However, the oscillation may be perceived at multiple splitting ASes. The different paths may pass through a different number of egress interfaces at which the mechanism is enforced. For example, path $\pi_{3}$ in Fig. 5(c) only passes through one critical egress interface (at AS $A_{0}$ ), whereas paths $\pi_{1}$ and $\pi_{2}$ pass through two critical egress interfaces. Conversely, each path in Fig. 5(d) passes through two egress interfaces at which a load-balancing mechanism is enforced. Any stabilization mechanism may thus be applied repeatedly and with different frequency to flows belonging to the same oscillation-prone system.

In the intra-domain context, the mechanism-enforcement architecture envisages a centralized oscillation-suppression service (OSS) in each AS. The OSS is capable of interacting with the border routers at the egress interfaces. For a splitting AS, this OSS functions as displayed in Fig. 6. By collecting aggregate load statistics from the border routers, the OSS in the splitting AS can identify the egress interfaces between which oscillation occurs (through correlation). As the presence of such oscillation means that the AS is obliged to enforce a stabilization mechanism, the OSS equips every oscillationperceiving border router $r_{i}$ with data $M_{i}$ that is necessary to enforce the mechanism (e.g., start time of the next interval). By further collecting load statistics from the egresses, the OSS monitors and continuously adapts the execution of the mechanism. The border routers communicate with the origins of the oscillating flows by appending mechanism-relevant information to passing packets. 


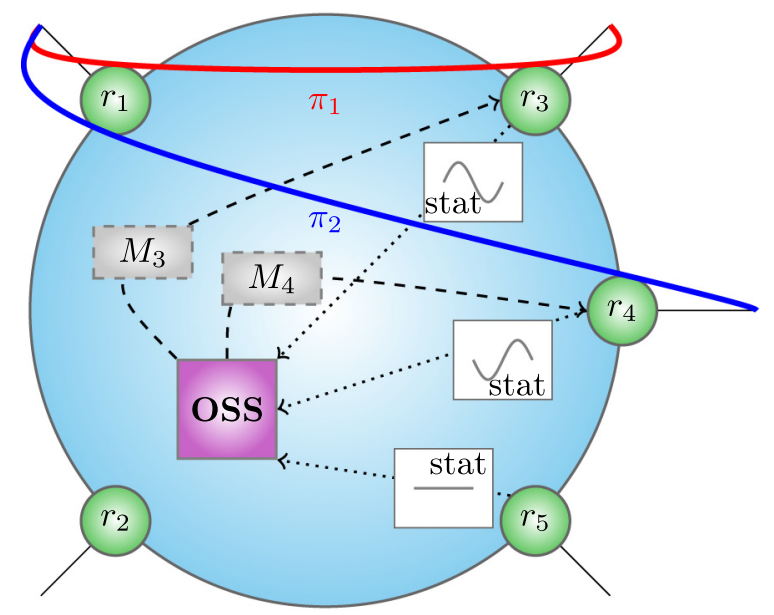

Fig. 6. Mechanism-enforcement architecture (within the splitting AS).

\subsection{FLOSS in practice}

In the following, we discuss how the FLOSS mechanism could be applied by the mechanism-enforcement architecture from Section 7.2, while conforming to the practicality requirements laid out in Section 7.1, namely limited overhead and coordination-freeness.

\subsubsection{Limited overhead}

Registration on routers. In order to signal that end-hosts must register for an upcoming time interval, a border router appends the start time $t_{i}$ of the next interval to passing packets. If an end-host witnesses such a call for registrations in its packets, it can send a packet with a registration request over the desired egress. A border router can keep track of registrations using a Bloom filter, which approximates a set of flow IDs. A Bloom filter offers constant complexity for both lookup and insertion, although suffering from false positives. When checking for registrations, false positives result in unregistered flows being able to send over an egress and being rewarded like loyal flows. However, the enforced migration rate $\rho$ can simply be discounted by the false-positive rate of the Bloom filter such that the desired migration rate is enforced despite the presence of lucky unregistered flows.

Enforcement of single registration. In order to avoid that an end-host registers on multiple egresses, a border router forwards all registrations to the OSS, which keeps track of egress-specific registration by flows and can therefore spot multiple registrations by the same flow. If multiple registrations are detected, the OSS pushes a blacklist update for the malicious flow ID to the border routers. In order to avoid introducing DoS attacks where a malicious actor provokes the blacklisting of an end-host by sending multiple registrations, we assume some form of lightweight source authentication, which is typically offered by path-aware Internet architectures [34].

Selective admission of migrating flows. Border routers need an efficient way to decide whether to grant registration applications to flows that are willing to switch paths, while preserving the property that a maximum share $\rho$ of flows migrates. Such selective admission can be implemented using a publicly know hash function $h$, which maps the flow ID $f$ to the interval $[0,1]$. If $h\left(t_{i} \mid f\right)<\rho$, the registration is granted, where $t_{i}$ is the beginning time of the next registrationenforcement interval. This construction has the advantage that an end-host can locally check whether it will be accepted on the alternative ingress, as $h, t_{i}$, and $f$ are known to the end-host. Therefore, the border router is not bothered by registration requests from end-hosts that would be rejected. Furthermore, it is important to choose the flow ID $f$ based on attributes that the source end-host cannot easily influence without compromising its communication, e.g., source and destination IP, but not source or destination port.

Small traffic allowance for unregistered flows. While unregistered end-hosts should not be able to properly use an egress, these end-hosts should be able to send a few packets over the egress to measure the latency of the corresponding path. Also, short flows, e.g., DNS requests, should not be required to obtain a registration. Such a limited traffic allowance can be efficiently achieved by applying the mechanism only to a subset of packets, e.g., by sub-sampling. If registrations are only checked for a sub-set of packets, even an unregistered flow has a high chance of getting a few packets through the egress, while still experiencing severe disruption when sending a large number of packets over the egress. Due to the structure of congestion-control algorithms, sub-sampling rates as low as $1 \%$ already cause enough packet drops to make a path completely unusable for unregistered flows [35]. Moreover, sub-sampling reduces the workload on border routers. 
Addition of new flows. In reality, new flows appear during the execution of the mechanism. Clearly, these flows cannot register in advance for an enforcement interval, as these flows do not exist beforehand. Therefore, new flows are also allowed to register at one path of their choice during an enforcement interval. In order to distinguish new flows from flows that merely pretend to be new, the FLOSS mechanism samples the active flows at both egresses in every interval and inserts them into a Bloom filter. These previously active flows are supposed to have a registration in the subsequent interval. In contrast, truly new flows can be identified with a lookup failure in the mentioned Bloom filter. Due to false positives, a truly new flow might be mistaken for a previously active flow and thus be denied a retroactive registration. However, given a small false-positive probability, the probability that such a mistake appears at multiple egresses is negligible such that registration at one path should always be possible in practice. As all new flows (except the falsepositive new flows) during an interval must be expected to flock to the cheaper path, the migration allowance must be discounted by the birth rate of flows.

\subsubsection{Coordination-freeness}

If there is one splitting AS for an oscillation-prone system, there are no unintended effects due to distributed application of the mechanism. However, as explained in Section 7.2, there may be multiple mechanism-enforcing ASes along a path. If $n_{i}$ is the number of splitting ASes along path $\pi_{i}$, the costs for obtaining a registration for $\pi_{i}$ and for using $\pi_{i}$ without a registration are $n_{i} \cdot c_{\mathrm{a}}$ and $n_{i} \cdot c_{\mathrm{p}}$, respectively. In cases where $n_{i}$ is the same for every path $\pi_{i}$ of an oscillation pattern (such as in Fig. 5(d)), the incentives for the end-hosts thus do not change compared to a single-application scenario. However, if $n_{i}$ is different for the paths $\pi_{i}$ in the oscillation-prone system (such as in Fig. 5(c)), the registration cost for different paths may be different. For example, the registration cost for obtaining a registration of path $\pi_{3}$ in Fig. $5(\mathrm{c})$ is $c_{\mathrm{a}}$, whereas the corresponding cost for paths $\pi_{1}$ and $\pi_{2}$ is $2 c_{\mathrm{a}}$. Since $c_{\mathrm{p}}=\infty>n_{i} c_{\mathrm{a}}$ for all finite $n_{i}$, registering for a path is still worthwhile. However, an equilibrium between the two egresses of AS $A_{0}$ is only reached if $\left(f_{\pi_{1}}+f_{\pi_{2}}\right)^{p}+2 c_{\mathrm{a}}=f_{\pi_{3}}^{p}+c_{\mathrm{a}}$, which implies stability at unequal load. However, since the cost $c_{\mathrm{a}}$ for obtaining a registration is modest (just a single packet as explained in Section 7.3.1), the resulting load imbalance between the ASes is also modest. Therefore, no explicit inter-AS coordination is needed.

\subsection{CROSS in practice}

In this section, we discuss the CROSS mechanism with respect to the two practicality requirements.

\subsubsection{Limited overhead}

Compared to FLOSS, the only additional piece of functionality needed for CROSS is puzzle verification. Efficient puzzlesolution verification on border routers is performed by a hash function evaluation with the appropriate arguments, among which is the solution value provided by the data packet (cf. Section 6.2).

\subsubsection{Coordination-freeness}

Like FLOSS, CROSS suffers from the minor issue that some paths may require more registrations than other paths. Concerning backup registrations, multiple applications of the mechanism do not constitute a problem, as an end-host always has to solve only one puzzle to obtain a backup registration. For example, an end-host in the network of Fig. 5(c) could insure against path failure as follows. At AS $A_{0}$, the end-host would obtain a normal registration for $\pi_{3}$ and a backup registration for $\pi_{1}$ and $\pi_{2}$. Such a combined backup registration is possible by including only the respective egress of AS $A_{0}$ in the puzzle solution, not the specific path. At AS $A_{1}$, the end-host can then obtain a normal registration for one of these paths, e.g., $\pi_{1}$. If the end-host desires an additional insurance against failure of path $\pi_{1}$, the end-host can solve a puzzle to obtain a backup registration for $\pi_{2}$ at AS $A_{1}$. Since only one puzzle per backup path is needed, no explicit inter-AS coordination is necessary to preserve the incentives of the CROSS mechanism.

\section{Related work}

Prior research has devised traffic-engineering tools to improve network stability. However, due to the traditional paradigm of network-controlled path selection, most tools assume that packet forwarding is performed by series of decisions taken by the hops along a path. Systems such as AMP [11], ReplEx [10], Homeostasis [12], and HALO [13] thus prescribe how routers along a path should take forwarding decisions, mostly by adapting traffic-splitting ratios based on network information. If packets must be forwarded along a path chosen by the end-host, these schemes cannot be used.

An alternative line of work is generally compatible with the emerging paradigm of end-point path selection. Assuming source routing, this flavor of research prescribes path-selection strategies that lead to convergence. However, such convergent path-selection strategies are always designed for an intra-domain context, i.e., for path selection within a domain where end-points are under control of the network operator. Due to the selfishness of end-hosts in the interdomain context, these schemes are thus impractical. For example, Proportional Sticky Routing [18] relies on self-restraint of end-points, which leads to persistent preference of shortest paths over alternative paths even when alternative paths are more attractive. The convergence of MATE [14] and the rerouting strategy designed by Kelly and Voice [17] is built on the assumption that the end-points restrain themselves to a maximum speed when reallocating traffic on cheaper paths, 
which cannot be expected from selfish end-hosts. In TeXCP [16], end-points are expected to comply with maximum traffic-reallocation allowances dynamically set by the network. Similarly, the rerouting policies designed by Fischer and Vöcking [8] require that end-hosts do not exceed a certain probability for switching to a cheaper path. Finally, OPS [15] also demands behavior from end-hosts that is irrational in a game-theoretic sense, in particular the probabilistic usage of sub-optimal paths.

Inter-domain traffic engineering by means of incentives has only been studied in context of the BGP ecosystem, thus not accounting for path choice by end-hosts. Given rational ASes, there are different methods to achieve stability for inter-domain traffic: incentive-compatible yet oscillation-free BGP policies [36,37], egress-router selection under QoS constraints [38], cooperative traffic-engineering agreements between ASes reached by Nash bargaining [39], and the use of prices as traffic-steering incentives [40].

\section{Conclusion}

In this work, we have set up a game-theoretic framework that allows to test path-selection strategies on their viability for selfish end-hosts, i.e., to show whether it is rational for an end-host to adopt a path-selection strategy, given that all other end-hosts use said path-selection strategy. Only strategies that form such equilibria may be adopted in an Internet environment, where end-hosts are self-interested and uncontrolled.

Using this framework, we have shown that the non-oscillatory path-selection strategies traditionally proposed in the literature are not rational strategies and thus cannot be expected to be adopted by selfish, unrestricted end-hosts. This insight suggests that end-hosts must be incentivized to abstain from oscillatory path selection by means of stabilization mechanisms. We have designed two stabilization mechanisms and proved their incentive compatibility.

We understand our work as a first step and we believe that it opens several interesting avenues for future research. In particular, it would be interesting to quantify the cost of oscillation to a network and to investigate its relationship to the network type. Comparing the oscillation cost to the overhead of stabilization mechanisms would then allow to characterize the conditions under which the employment of stabilization mechanisms is appropriate.

\section{Declaration of competing interest}

The authors declare that they have no known competing financial interests or personal relationships that could have appeared to influence the work reported in this paper.

\section{Acknowledgments}

We gratefully acknowledge support from ETH Zurich, from the Zurich Information Security and Privacy Center (ZISC), Switzerland, from SNSF, Switzerland for project ESCALATE (200021L182005), and from WWTF, Austria for project WHATIF (ICT19-045, 2020-2024).

\section{References}

[1] D. Andersen, H. Balakrishnan, F. Kaashoek, R. Morris, Resilient overlay networks, in: Proceedings of the ACM Symposium on Operating Systems Principles, SOSP '01, 2001.

[2] B. Raghavan, A.C. Snoeren, A system for authenticated policy-compliant routing, ACM SIGCOMM Comput. Commun. Rev. 34 (4) (2004).

[3] W. Xu, J. Rexford, MIRO: Multi-path interdomain routing, in: Proceedings of the ACM SIGCOMM Conference, SIGCOMM '06, 2006.

[4] P.B. Godfrey, I. Ganichev, S. Shenker, I. Stoica, Pathlet routing, ACM SIGCOMM Comput. Commun. Rev. 39 (4) (2009) 111-122.

[5] C. Filsfils, N.K. Nainar, C. Pignataro, J.C. Cardona, P. Francois, The segment routing architecture, in: 2015 IEEE Global Communications Conference (GLOBECOM), 2015.

[6] D. Barrera, L. Chuat, A. Perrig, R.M. Reischuk, P. Szalachowski, The SCION internet architecture, Commun. ACM 60 (6) (2017).

[7] S. Dawkins, Path Aware Networking: Obstacles to Deployment (A Bestiary of Roads Not Taken), draft-irtf-panrg-what-not-to-do-07, Internet Engineering Task Force, 2020, Work in Progress. https://datatracker.ietf.org/doc/html/draft-irtf-panrg-what-not-to-do-07.

[8] S. Fischer, B. Vöcking, Adaptive routing with stale information, Theoret. Comput. Sci. 410 (36) (2009) 3357-3371.

[9] A. Shaikh, J. Rexford, K.G. Shin, Evaluating the impact of stale link state on quality-of-service routing, IEEE/ACM Trans. Netw. 9 (2) (2001) $162-176$.

[10] S. Fischer, N. Kammenhuber, A. Feldmann, REPLEX: Dynamic traffic engineering based on Wardrop routing policies, in: Proceedings of the 2006 ACM CoNEXT Conference, ACM, 2006, p. 1.

[11] I. Gojmerac, T. Ziegler, F. Ricciato, P. Reichl, Adaptive multipath routing for dynamic traffic engineering, in: GLOBECOM'03. IEEE Global Telecommunications Conference (IEEE Cat. No. 03CH37489), IEEE, 2003.

[12] A. Kvalbein, C. Dovrolis, C. Muthu, Multipath load-adaptive routing: Putting the emphasis on robustness and simplicity, in: 2009 17th IEEE International Conference on Network Protocols, IEEE, 2009.

[13] N. Michael, A. Tang, Halo: Hop-by-hop adaptive link-state optimal routing, IEEE/ACM Trans. Netw. 23 (6) (2014) $1862-1875$.

[14] A. Elwalid, C. Jin, S. Low, I. Widjaja, MATE: Multipath adaptive traffic engineering, Comput. Netw. 40 (6) (2002) 695-709.

[15] B. Jonglez, B. Gaujal, Distributed and adaptive routing based on game theory, in: 2017 29th International Teletraffic Congress (ITC 29), IEEE, 2017.

[16] S. Kandula, D. Katabi, B. Davie, A. Charny, Walking the tightrope: Responsive yet stable traffic engineering, in: ACM SIGCOMM Computer Communication Review, ACM, 2005.

[17] F. Kelly, T. Voice, Stability of end-to-end algorithms for joint routing and rate control, ACM SIGCOMM Comput. Commun. Rev. 35 (2) (2005) 5-12. 
[18] S. Nelakuditi, Z.-L. Zhang, R.P. Tsang, D.H.-C. Du, Adaptive proportional routing: a localized QoS routing approach, IEEE/ACM Trans. Netw. 10 (6) (2002) 790-804.

[19] J.G. Wardrop, Some theoretical aspects of road traffic research, Proc. Inst. Civ. Eng. 1 (3) (1952) 325-362.

[20] L. Qiu, Y.R. Yang, Y. Zhang, S. Shenker, On selfish routing in Internet-like environments, in: Proceedings of the 2003 Conference on Applications, Technologies, Architectures, and Protocols for Computer Communications, 2003.

[21] T. Roughgarden, The price of anarchy is independent of the network topology, J. Comput. System Sci. 67 (2) (2003) $341-364$.

[22] T. Roughgarden, É. Tardos, How bad is selfish routing?, J. ACM 49 (2) (2002) 236-259.

[23] G. Christodoulou, E. Koutsoupias, The price of anarchy of finite congestion games, in: Proceedings of the Thirty-Seventh Annual ACM Symposium on Theory of Computing, 2005.

[24] R. Holzman, N. Law-Yone, Strong equilibrium in congestion games, Games Econ. Behav. 21 (1-2) (1997) 85-101.

[25] R.W. Rosenthal, A class of games possessing pure-strategy Nash equilibria, Internat. J. Game Theory 2 (1) (1973) 65-67.

[26] S. Fischer, B. Vöcking, On the evolution of selfish routing, in: European Symposium on Algorithms, 2004.

[27] S. Fischer, B. Vöcking, Evolutionary game theory with applications to adaptive routing, in: European Conference on Complex Systems (ECCS), 2005.

[28] W.H. Sandholm, Potential games with continuous player sets, J. Econ. Theory 97 (1) (2001) 81-108.

[29] S. Scherrer, M. Legner, A. Perrig, S. Schmid, Incentivizing stable path selection in future internet architectures, 2020, arXiv:2009.12105 [cs.NI]

[30] S.A. Campbell, J. Belair, T. Ohira, J. Milton, Complex dynamics and multistability in a damped harmonic oscillator with delayed negative feedback, Chaos 5 (4) (1995) 640-645.

[31] T. Erneux, Applied Delay Differential Equations, Springer Science \& Business Media, 2009.

[32] A. Akella, S. Seshan, R. Karp, S. Shenker, C. Papadimitriou, Selfish behavior and stability of the internet: a game-theoretic analysis of TCP, ACM SIGCOMM Comput. Commun. Rev. 32 (4) (2002) 117-130.

[33] P. Godfrey, M. Schapira, A. Zohar, S. Shenker, Incentive compatibility and dynamics of congestion control, in: ACM SIGMETRICS Performance Evaluation Review, 2010.

[34] B. Rothenberger, D. Roos, M. Legner, A. Perrig, PISKES: Pragmatic internet-scale key-establishment system, in: Proceedings of the 15th ACM Asia Conference on Computer and Communications Security (ASIA CCS'20), 2020, http://dx.doi.org/10.1145/3320269.3384743.

[35] T. Lukaseder, L. Bradatsch, B. Erb, R.W. Van Der Heijden, F. Kargl, A comparison of TCP congestion control algorithms in 10G networks, in: 2016 IEEE 41st Conference on Local Computer Networks (LCN), IEEE, 2016, pp. 706-714.

[36] Y.R. Yang, H. Xie, H. Wang, A. Silberschatz, A. Krishnamurthy, Y. Liu, L.E. Li, On route selection for interdomain traffic engineering, IEEE Netw. (2005).

[37] J. Feigenbaum, V. Ramachandran, M. Schapira, Incentive-compatible interdomain routing, in: Proceedings of the 7th ACM Conference on Electronic Commerce, ACM, 2006.

[38] K.-H. Ho, G. Pavlou, M. Howarth, N. Wang, An Incentive-based quality of service aware algorithm for offline inter-AS traffic engineering, in: 2004 IEEE International Workshop on IP Operations and Management, 2004.

[39] G. Shrimali, A. Akella, A. Mutapcic, Cooperative interdomain traffic engineering using Nash bargaining and decomposition, IEEE/ACM Trans Netw. 18 (2) (2009) 341-352.

[40] R. Mortier, I. Pratt, Incentive based inter-domain routing, in: Group Communications and Charges. Technology and Business Models, Springer, 2003.

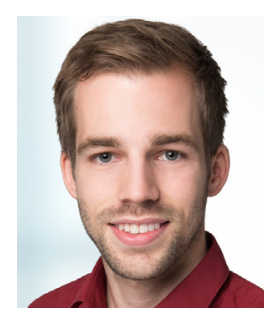

Simon Scherrer is a doctoral student in the Network Security Group at ETH Zurich, Switzerland, under the supervision of Professor Adrian Perrig. His research interests include game theory in networks, the investigation of network architectures with end-host path selection, and probabilistic traffic monitoring. He obtained an MSc degree from ETH Zurich in 2019.

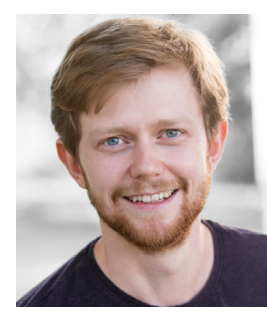

Markus Legner is a postdoctoral researcher in the Network Security Group headed by Prof. Dr. Adrian Perrig at ETH Zurich, Switzerland. He is working on SCION, a next-generation path-aware Internet architecture that at the same time provides high security and high availability. In particular, he focuses on improving the control-plane security and investigating effects of end-host-based path selection on network properties. Before joining the Network Security Group, Markus received his Ph.D. in theoretical physics from ETH Zurich in 2016.

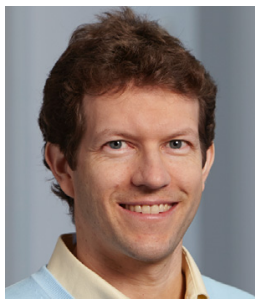

Adrian Perrig is a Professor at the Department of Computer Science at ETH Zürich, Switzerland, where he leads the network security group. He earned his MS and PhD degrees in Computer Science from Carnegie Mellon University, and spent three years during his $\mathrm{PhD}$ at the University of California at Berkeley. He received his BSc degree in Computer Engineering from EPFL. 


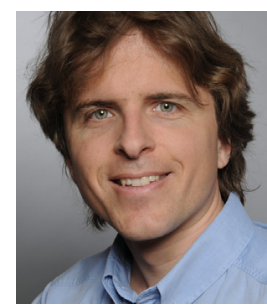

Stefan Schmid is a Full Professor at the University of Vienna, Austria. He completed his MSc and PhD at ETH Zurich, and he has worked as a Postdoc at TU Munich and University of Paderborn, as a Senior Research Scientist at T-Labs in Berlin, and as an Associate Professor at Aalborg University, Denmark. Stefan Schmid received the IEEE Communications Society ITC Early Career Award 2016 and an ERC Consolidator Grant 2019. 